Coherence-polarization properties of fields radiated from transversely periodic electromagnetic sources

This article has been downloaded from IOPscience. Please scroll down to see the full text article. 2013 J. Opt. 15055701

(http://iopscience.iop.org/2040-8986/15/5/055701)

View the table of contents for this issue, or go to the journal homepage for more

Download details:

IP Address: 147.96.14.15

The article was downloaded on 29/07/2013 at 13:26

Please note that terms and conditions apply. 


\title{
Coherence-polarization properties of fields radiated from transversely periodic electromagnetic sources
}

\author{
M Santarsiero ${ }^{1}$, J C G de Sande ${ }^{2}$, G Piquero ${ }^{3}$ and F Gori ${ }^{1}$ \\ ${ }^{1}$ Dipartimento di Ingegneria, and CNISM, Università Roma Tre, Via della Vasca Navale 84, I-00146 \\ Rome, Italy \\ ${ }^{2}$ Departamento de Circuitos y Sistemas, Universidad Politécnica de Madrid, E-28031 Madrid, Spain \\ ${ }^{3}$ Departamento de Óptica, Facultad de Ciencias Físicas, Universidad Complutense de Madrid, Ciudad \\ Universitaria, E-28040 Madrid, Spain
}

E-mail: santarsiero@fis.uniroma3.it

Received 3 January 2013, accepted for publication 18 February 2013

Published 20 March 2013

Online at stacks.iop.org/JOpt/15/055701

\begin{abstract}
Planar electromagnetic sources characterized by a periodic variation of their beam coherence-polarization matrix are investigated, as far as the polarization features of the radiated fields are concerned, within the framework of the paraxial approximation. A propagation scheme based on plane-wave decomposition leads to a longitudinal periodicity of the polarization properties of the field, thus extending the Talbot effect to the case of partially coherent electromagnetic sources. The polarization features of beams radiated from sources of this type are illustrated by means of simple examples. In particular, it is shown that completely unpolarized sources with uniform intensity profiles can be easily realized, for which the propagated field becomes perfectly polarized across some transverse planes, and vice versa.
\end{abstract}

Keywords: coherence, polarization, partially coherent electromagnetic sources, Talbot effect

(Some figures may appear in colour only in the online journal)

\section{Introduction}

It is well known that the transverse periodicity of a coherent scalar light field across a certain plane gives rise to longitudinal periodicities of the propagated field. This effect was first reported by Talbot in 1836 [1]. Since then, the so called Talbot effect, or self-imaging, has been the subject of much research, both theoretical and experimental, in various branches of optics [2-12]. A somewhat related phenomenon is the Lau effect, pertaining to the features of the field propagated from a completely incoherent light source having a transversely periodic intensity profile [13]. Such effects can be unified, within the framework of the coherence theory, if one considers the field propagated from a partially coherent planar source characterized by a transversely periodic second-order correlation function [14-21]. Most of the previous cited papers deal with scalar treatments. Only a few of them (see, for example, [4, 6, 8-10]) take the polarization of the field into account, but assuming perfectly deterministic fields. In such cases, even though the generated fields have periodic structure of the polarization state across the transverse plane, the degree of polarization remains equal to one everywhere, i.e., neither transverse nor longitudinal variations of the polarization degree are obtained.

In recent years the interest has grown in light sources that are both partially coherent and partially polarized, or that present nonuniform polarization characteristics. Within the paraxial approximation, a tool for dealing with such partially coherent electromagnetic sources is the $2 \times 2$ beam coherence-polarization (BCP) matrix [22], which collects the second-order correlation functions among all the transverse components of the electric field across the source plane. In particular, this tool, as well as its counterpart in the space-frequency domain [23], allows the 
effects of the coherence on the polarization properties of a paraxially propagating field to be investigated [24-41]. Sources with periodic BCP matrices can be obtained, for instance, when partially coherent fields are diffracted by periodical structures, such as amplitude, phase or polarization gratings (PGs) [42-44]. The case of perfectly coherent radiation diffracted by polarization gratings has already been studied, e.g., in [9]. As we shall see in the following, another way to obtain transversely periodic partially coherent electromagnetic sources is by means of pseudo-depolarizers that use anisotropic crystal wedges [45].

In this paper we consider planar electromagnetic sources with periodic BCP matrices and study the polarization characteristics of the radiated field, thus extending the results already obtained in the scalar domain and obtaining more general results for the vectorial case. While some of the achieved issues, such as the longitudinal periodicity of the field at Talbot distances, have a scalar counterpart, some of them, namely the ones pertinent to the propagation of the Stokes parameters and the polarization degree, are intrinsically vectorial.

The paper is structured as follows. In section 2 the formalism is recalled and the BCP matrix of a periodic electromagnetic source, together with its main features, is introduced. The characteristics of the field propagated from such a source are presented in section 3, while some significant examples are shown in section 4. The main conclusions are summarized in section 5.

\section{Transversely periodic electromagnetic sources}

We consider secondary planar sources located across the plane $z=0$ of a rectangular reference frame $(x y z)$. For simplicity, we limit ourselves to sources that depend only on one of the transverse coordinates (say, $x$ ) so that the problem can be considered as one-dimensional, but the results we are going to obtain can be easily extended to the two-dimensional case. Therefore, we shall always omit the dependence on the $y$ coordinate.

Within the framework of the paraxial approximation, one can account for the complete set of space-time correlation functions at two typical points $x_{1}$ and $x_{2}$ of the source by using the BCP matrix [22], which is defined as the correlation matrix of the Jones vectors of the field at the points $x_{1}$ and $x_{2}$. More precisely, if the Jones vector of the electric field at the point $(x, 0)$ is introduced, i.e.,

$$
\mathcal{E}(x, 0)=\left(\begin{array}{l}
\mathcal{E}_{x}(x, 0) \\
\mathcal{E}_{y}(x, 0)
\end{array}\right),
$$

where the involved quantities are random variables of time, the corresponding BCP matrix is defined as

$$
\begin{aligned}
\hat{J}\left(x_{1}, x_{2}, 0\right) & =\left\langle\mathcal{E}\left(x_{1}, 0\right) \mathcal{E}^{\dagger}\left(x_{2}, 0\right)\right\rangle \\
& =\left(\begin{array}{ll}
J_{x x}\left(x_{1}, x_{2}, 0\right) & J_{x y}\left(x_{1}, x_{2}, 0\right) \\
J_{y x}\left(x_{1}, x_{2}, 0\right) & J_{y y}\left(x_{1}, x_{2}, 0\right)
\end{array}\right),
\end{aligned}
$$

with the dagger denoting Hermitian conjugation and the angle brackets time average. Therefore, the elements of the BCP matrix give the cross-correlation between the $\alpha$ - and $\beta$ components $(\alpha, \beta=x, y)$ of the electric field at points $x_{1}$ and $x_{2}$ for zero time delay. In the case of polychromatic fields, use should be made of the cross-spectral density tensor defined in the spectral domain [23,46], but the two definitions are equivalent if quasi-monochromatic sources are considered. Since we limit ourselves to quasi-monochromatic sources, we shall continue using the definition in equation (2), but our results could be easily transposed to the space-frequency domain. It should be kept in mind that, in order for a $2 \times 2$ matrix to represent the BCP matrix of a physically realizable source, the non-negativity condition has to be fulfilled [22, 46].

The local properties of the source are specified by the BCP matrix with $x_{1}=x_{2}=x$, which coincides with the polarization matrix $\hat{P}(x, 0)$ [46]. In fact, the whole set of Stokes parameters for the radiation can be evaluated starting from the values of the elements of the polarization matrix as follows [46]:

$$
\mathbf{s}(x, 0)=\left(\begin{array}{c}
s_{0}(x, 0) \\
s_{1}(x, 0) \\
s_{2}(x, 0) \\
s_{3}(x, 0)
\end{array}\right)=\left(\begin{array}{c}
P_{x x}(x, 0)+P_{y y}(x, 0) \\
P_{x x}(x, 0)-P_{y y}(x, 0) \\
2 \operatorname{Re}\left[P_{y x}(x, 0)\right] \\
2 \operatorname{Im}\left[P_{y x}(x, 0)\right]
\end{array}\right),
$$

with $\mathrm{Re}$ and $\mathrm{Im}$ denoting the real and imaginary parts, respectively. In particular, the optical intensity of the source and its degree of polarization at the coordinate $x$ turn out to be

$$
I(x, 0)=s_{0}(x, 0)=\operatorname{Tr}[\hat{P}(x, 0)]
$$

and

$$
p(x, 0)=\sqrt{1-\frac{4 \operatorname{Det}[\hat{P}(x, 0)]}{\{\operatorname{Tr}[\hat{P}(x, 0)]\}^{2}}},
$$

respectively, where Det stands for determinant and $\operatorname{Tr}$ for trace.

We want to study the propagation properties of the most general BCP matrix endowed with the property of being spatially periodic across the source plane. On denoting the period by $L$, each of the elements of $\hat{J}\left(x_{1}, x_{2}, 0\right)$ can be written according to a Fourier expansion of the form

$$
J_{\alpha \beta}\left(x_{1}, x_{2}, 0\right)=\sum_{n, m=-\infty}^{\infty} a_{n m}^{\alpha \beta} \exp \left[\mathrm{i} \frac{2 \pi}{L}\left(n x_{1}-m x_{2}\right)\right]
$$

(with $\alpha, \beta=x, y$ ), where the expansion coefficients are evaluated as

$$
\begin{aligned}
a_{n m}^{\alpha \beta}= & \frac{1}{L^{2}} \int_{-L / 2}^{L / 2} \int_{-L / 2}^{L / 2} J_{\alpha \beta}\left(x_{1}, x_{2}, 0\right) \\
& \times \exp \left[-\mathrm{i} \frac{2 \pi}{L}\left(n x_{1}-m x_{2}\right)\right] \mathrm{d} x_{1} \mathrm{~d} x_{2} .
\end{aligned}
$$

It is convenient to introduce the $2 \times 2$ matrices $\hat{A}_{n m}=$ $\left\{a_{n m}^{\alpha \beta}\right\}$, so that the BCP matrix in equation (2) can be written as

$$
\hat{J}\left(x_{1}, x_{2}, 0\right)=\sum_{n, m=-\infty}^{\infty} \hat{A}_{n m} \exp \left[\mathrm{i} \frac{2 \pi}{L}\left(n x_{1}-m x_{2}\right)\right] .
$$


From its definition, it turns out at once that $\hat{A}_{m n}=$ $\hat{A}_{n m}^{\dagger}$. Further conditions on the matrices $\hat{A}_{n m}$, ensuring the non-negativeness of $\hat{J}\left(x_{1}, x_{2}, 0\right)$, will be made clear in section 3 .

The local polarization properties of the source are derived from the expression of the BCP matrix on letting $x_{1}=x_{2}=x$, through the polarization matrix, that is

$$
\hat{P}(x, 0)=\sum_{n, m=-\infty}^{\infty} \hat{A}_{n m} \exp \left[\mathrm{i} \frac{2 \pi x}{L}(n-m)\right] .
$$

\section{The propagated field}

In order to study the effects of the propagation on the field radiated from the periodic source, we adopt a superposition scheme involving a discrete set of plane waves, suitably tilted and polarized, and suitably correlated with one another.

Let us denote by $\mathbf{E}_{n}$, with $n$ an integer, the Jones vector of a typical plane wave in this set, and let $2 \pi n / L$ be the $x$ component of its wavevector. The components of $\mathbf{E}_{n}$ (denoted by $E_{x, n}$ and $E_{y, n}$ ) give the $x$ and $y$ components of the electric field of that wave at $x=0$ and $z=0$ and are, in general, random variables. On superimposing a virtually infinite number of such plane waves, the total field across the plane $z=0$ turns out to be

$$
\mathcal{E}(x, 0)=\sum_{n=-\infty}^{\infty} \mathbf{E}_{n} \exp \left[\mathrm{i} \frac{2 \pi}{L} n x\right],
$$

giving rise, according to equation (2), to the following $\mathrm{BCP}$ matrix:

$\hat{J}\left(x_{1}, x_{2}, 0\right)=\sum_{n, m=-\infty}^{\infty}\left\langle\mathbf{E}_{n} \mathbf{E}_{m}^{\dagger}\right\rangle \exp \left[\mathrm{i} \frac{2 \pi}{L}\left(n x_{1}-m x_{2}\right)\right]$.

On comparing equations (8) and (11) it is apparent that the Fourier coefficients $\hat{A}_{n m}$ give account of the correlations between all the field components of the $n$th and $m$ th plane waves in the expansion, that is

$$
\hat{A}_{n m}=\left\langle\mathbf{E}_{n} \mathbf{E}_{m}^{\dagger}\right\rangle=\left(\begin{array}{cc}
\left\langle E_{x, n} E_{x, m}^{*}\right\rangle & \left\langle E_{x, n} E_{y, m}^{*}\right\rangle \\
\left\langle E_{y, n} E_{x, m}^{*}\right\rangle & \left\langle E_{y, n} E_{y, m}^{*}\right\rangle
\end{array}\right) .
$$

It can be noted that the matrices with $n=m$ coincide with the polarization matrices of each of the plane waves, while the ones with $n \neq m$ give account of the correlations existing between the field components among different plane waves. This means that the matrices $\hat{A}_{n n}$ must be Hermitian, non-negative definite and have non-negative diagonal elements [46]. More generally, for all matrices, the Schwarz inequality has to be satisfied, thus implying

$$
\left|a_{n m}^{\alpha \beta}\right|^{2} \leq a_{n n}^{\alpha \alpha} a_{m m}^{\beta \beta} .
$$

Such a superposition scheme allows for the BCP matrix of the field propagated from the source to be evaluated by simply considering the free propagation of the field in equation (10). Within the paraxial approximation, the latter turns out to be

$$
\begin{aligned}
\mathcal{E}(x, z)= & \mathrm{e}^{\mathrm{i} k z} \sum_{n=-\infty}^{\infty} \mathbf{E}_{n} \exp \left[\mathrm{i} \frac{2 \pi}{L} n x\right] \\
& \times \exp \left[-\mathrm{i} 2 \pi \frac{\lambda n^{2} z}{2 L^{2}}\right],
\end{aligned}
$$

and, according to the Talbot effect, periodically reproduces during propagation with longitudinal periodicity given by $z_{\mathrm{T}}=2 L^{2} / \lambda$.

The BCP matrix at a distance $z$ from the source is finally obtained as

$$
\begin{aligned}
\hat{J}\left(x_{1}, x_{2}, z\right)= & \left\langle\mathcal{E}\left(x_{1}, z\right) \mathcal{E}^{\dagger}\left(x_{2}, z\right)\right\rangle \\
= & \sum_{n, m=-\infty}^{\infty} \hat{A}_{n m} \exp \left[\mathrm{i} \frac{2 \pi}{L}\left(n x_{1}-m x_{2}\right)\right] \\
& \times \exp \left[-\mathrm{i} \frac{2 \pi}{z_{\mathrm{T}}}\left(n^{2}-m^{2}\right) z\right] .
\end{aligned}
$$

\subsection{Propagation-invariant BCP matrices}

It is seen from equation (15) that, whatever the explicit form of the BCP matrix, there is one part of it that does not change upon propagation. Clearly, it is that part for which the difference $n^{2}-m^{2}$ vanishes, i.e., the sum of terms having $m= \pm n$. In particular, the BCP may contain only such terms. We shall say that in these cases the $\mathrm{BCP}$ is propagation invariant. Let us dwell on such fields for a while.

It is seen from equation (8) that $\hat{J}\left(x_{1}, x_{2}, 0\right)$ becomes the sum of a function of $x_{1}-x_{2}$ and a function of $x_{1}+x_{2}$. Accordingly, we let

$$
\hat{J}\left(x_{1}, x_{2}, 0\right)=\hat{J}_{M}\left(x_{1}-x_{2}\right)+\hat{J}_{P}\left(x_{1}+x_{2}\right),
$$

where

$$
\hat{J}_{M}\left(x_{1}-x_{2}\right)=\sum_{n=-\infty}^{\infty} \hat{A}_{n n} \exp \left[\mathrm{i} \frac{2 \pi}{L} n\left(x_{1}-x_{2}\right)\right]
$$

and

$$
\hat{J}_{P}\left(x_{1}+x_{2}\right)=\sum_{n=-\infty}^{\infty^{\prime}} \hat{A}_{n,-n} \exp \left[\mathrm{i} \frac{2 \pi}{L} n\left(x_{1}+x_{2}\right)\right],
$$

where the prime sign in the last sum means that the term with $n=0$ is missing.

We shall first consider the case in which only $\hat{J}_{M}$ survives. Then, the BCP becomes transversely shift invariant. This is a case in which a simple experimental procedure can be used to synthesize the field. In fact, the vectorial form of the van Cittert-Zernike theorem [24] applies and the field can be produced by a suitable set of partially polarized point-like sources mutually uncorrelated with each other and set in the input focal plane of a lens. Note also that the associated intensity, namely

$$
I_{\mathrm{u}}=\operatorname{Tr}\left\{\hat{J}_{M}(0)\right\}=\sum_{n=-\infty}^{\infty}\left(a_{n n}^{x x}+a_{n n}^{y y}\right)
$$


(where the suffix u stands for uncorrelated), is also uniform across a typical transverse plane. The same considerations hold for the three remaining Stokes parameters. From the definition in equation (3), in fact, they are given by

$$
\begin{aligned}
& s_{1}^{(\mathrm{u})}=\sum_{n=-\infty}^{\infty}\left(a_{n n}^{x x}-a_{n n}^{y y}\right), \\
& s_{2}^{(\mathrm{u})}=\sum_{n=-\infty}^{\infty}\left(a_{n n}^{x y}+a_{n n}^{y x}\right), \\
& s_{3}^{(\mathrm{u})}=\sum_{n=-\infty}^{\infty} \mathrm{i}\left(a_{n n}^{x y}-a_{n n}^{y x}\right),
\end{aligned}
$$

and turn out to be uniform.

Let us pass to the case in which also the second term in the right-hand side of equation (16) gives a contribution to the BCP. This occurs when there exists some correlation between the fields generated by those point-like sources that are symmetrically located with respect to that of zero order. Even if this requires a slightly more sophisticated experimental setup than the one needed in the previous case, single pair correlations are easily realized [47].

Notice that, at variance with the previous case, the intensity now varies with $x$ in a typical transverse plane, according to the law

$$
\begin{aligned}
I_{\mathrm{c}}(x) & =\operatorname{Tr}\left\{\hat{J}_{M}(0)+\hat{J}_{P}(2 x)\right\} \\
& =I_{\mathrm{u}}+\operatorname{Tr}\left\{\sum_{n=-\infty}^{\infty^{\prime}} \hat{A}_{n,-n} \exp \left[\mathrm{i} \frac{4 \pi}{L} n x\right]\right\}
\end{aligned}
$$

(where c stands for correlated) derived from equations (16)-(18) on letting $x_{1}=x_{2}=x$, because an interference term now appears in the intensity profile. On using the definition of the $\hat{A}_{n m}$ matrices, and their property of being $\hat{A}_{m n}=\hat{A}_{n m}^{\dagger}$, the above intensity can be written as

$$
\begin{aligned}
I_{\mathrm{c}}(x)= & I_{\mathrm{u}}+2 \sum_{n=1}^{\infty}\left[\left|a_{n,-n}^{x x}\right| \cos \left(\frac{4 \pi}{L} n x+\delta_{n}^{x x}\right)\right. \\
& \left.+\left|a_{n,-n}^{y y}\right| \cos \left(\frac{4 \pi}{L} n x+\delta_{n}^{y y}\right)\right],
\end{aligned}
$$

where the argument $\delta_{n}^{\alpha \beta}$ (with $\alpha, \beta=x, y$ ) of the complex quantity $a_{n,-n}^{\alpha \beta}$ has been introduced.

Even in this case, the evaluation of the Stokes parameters across a transverse plane leads to analogous results and an interference term appears for each of the three parameters [48], related to the correlation between Fourier components with opposite indices. In particular, we have

$$
\begin{aligned}
& s_{1}^{(\mathrm{c})}(x)=s_{1}^{(\mathrm{u})}+2 \sum_{n=1}^{\infty}\left[\left|a_{n,-n}^{x x}\right| \cos \left(\frac{4 \pi}{L} n x+\delta_{n}^{x x}\right)\right. \\
& \left.-\left|a_{n,-n}^{y y}\right| \cos \left(\frac{4 \pi}{L} n x+\delta_{n}^{y y}\right)\right], \\
& s_{2}^{(\mathrm{c})}(x)=s_{2}^{(\mathrm{u})}+2 \sum_{n=1}^{\infty}\left[\left|a_{n,-n}^{x y}\right| \cos \left(\frac{4 \pi}{L} n x+\delta_{n}^{x y}\right)\right. \\
& \left.+\left|a_{n,-n}^{y x}\right| \cos \left(\frac{4 \pi}{L} n x+\delta_{n}^{y x}\right)\right],
\end{aligned}
$$

$$
\begin{gathered}
s_{3}^{(\mathrm{c})}(x)=s_{3}^{(\mathrm{u})}-2 \sum_{n=1}^{\infty}\left[\left|a_{n,-n}^{x y}\right| \sin \left(\frac{4 \pi}{L} n x+\delta_{n}^{x y}\right)\right. \\
\left.-\left|a_{n,-n}^{y x}\right| \sin \left(\frac{4 \pi}{L} n x+\delta_{n}^{y x}\right)\right] .
\end{gathered}
$$

\subsection{Self-imaging of BCP matrices}

Let us now pass to more general situations. As can be deduced at once from the result in equation (15), in paraxial conditions the BCP matrix (and then all the polarization features) of the propagated field presents the same periodicity as a coherent transversely periodic field. In particular, it exactly reproduces at longitudinal coordinates equal to multiples of the Talbot distance $z_{\mathrm{T}}$. Moreover, the same profile, but transversely shifted by a half-period, is observed across all planes halfway between two adjacent Talbot planes. The first property directly comes from the fact that, for $z=z_{\mathrm{T}}$, the last exponential in equation (15) is unitary for all choices of the integers $n$ and $m$. To obtain the second property, it is sufficient to note that, for $z=z_{\mathrm{T}} / 2$, the above exponential becomes

$$
\begin{aligned}
\exp \left[-\mathrm{i} \pi\left(n^{2}-m^{2}\right)\right] & =(-1)^{n^{2}}(-1)^{m^{2}}=(-1)^{n}(-1)^{m} \\
& =\exp [-\mathrm{i} \pi(n-m)]
\end{aligned}
$$

so that equation (15) can be written as

$$
\begin{aligned}
& \hat{J}\left(x_{1}, x_{2}, z_{\mathrm{T}} / 2\right)=\sum_{n, m=-\infty}^{\infty} \hat{A}_{n m} \\
& \quad \times \exp \left\{\mathrm{i} \frac{2 \pi}{L}\left[n\left(x_{1}-\frac{L}{2}\right)-m\left(x_{2}-\frac{L}{2}\right)\right]\right\} .
\end{aligned}
$$

Further interesting properties concern the behavior of the $\mathrm{BCP}$ matrix at shorter distances. For instance, for $z=z_{\mathrm{T}} / 4$ the exponential term depending on $z$ in equation (15) becomes

$$
\exp \left[-\mathrm{i} \frac{\pi}{2}\left(n^{2}-m^{2}\right)\right]=(-\mathrm{i})^{n^{2}} \mathrm{i}^{m^{2}}=(-\mathrm{i})^{v} \mathrm{i}^{\mu}=\mathrm{i}^{\mu-v},
$$

where the integers $v$ and $\mu$ represent the parities of $n$ and $m$, respectively, i.e., $v(\mu)=0$ if $n(m)$ is even, while $v(\mu)=1$ if $n(m)$ is odd. In particular, if the Fourier expansion of the BCP matrix contains only even or only odd indices, all exponential terms turn out to be unity, and the BCP matrix exactly reproduces itself. This, of course, is a consequence of the fact that in such a case the original BCP matrix is periodic with period $L / 2$. A slightly less obvious result is that, if the $\mathrm{BCP}$ matrix contains only odd terms, it also reproduces at $z=z_{\mathrm{T}} / 8$. This behavior, already noticed in the case of perfectly coherent vectorial fields [9], comes from the fact that, if both $n$ and $m$ are odd numbers, we can set $n=2 \ell+1$ and $m=2 j+1$ ( $\ell, j$ integers $)$, and the exponential depending on $z$ in equation (15) turns out to be

$$
\begin{aligned}
\exp & {\left[-\mathrm{i} \frac{\pi}{4}\left(n^{2}-m^{2}\right)\right] } \\
& =\exp \left\{-\mathrm{i} \frac{\pi}{4}\left[(2 \ell+1)^{2}-(2 j+1)^{2}\right]\right\} \\
& =(-1)^{\ell(\ell+1)}(-1)^{j(j+1)}=1 .
\end{aligned}
$$


Further properties of the propagated $\mathrm{BCP}$ matrix are hardly envisaged in the most general case but, as we shall see in the following, interesting features can be observed for some specific forms of the BCP matrix across the source.

\section{Examples}

\subsection{Two component plane waves}

To give an example of a case in which only terms with $n= \pm m$ are present in the expansion of the BCP matrix, we consider that only two plane waves contribute to the total field. Such waves, in fact, can always be considered as the orders +1 and -1 of a suitable set of plane waves. From the general result in section 3, it follows that the BCP matrix of the radiated field is propagation invariant, regardless of the correlation existing between the two plane waves and their polarization states. We can take, for instance, two waves carrying the same power and perfectly polarized, one of them propagating as the order +1 and linearly polarized at $\pi / 4$ with respect to the $x$ axis, the other one directed as the order -1 and linearly polarized along the $y$ axis.

If the two waves are mutually uncorrelated, as can be easily verified, the BCP matrix of the radiated field turns out to be

$$
\begin{aligned}
\hat{J}\left(x_{1},\right. & \left.x_{2}, z\right) \\
& =\hat{J}\left(x_{1}, x_{2}, 0\right)=\hat{J}_{M}\left(x_{1}-x_{2}\right) \\
& =\frac{I_{0}}{4}\left(\begin{array}{cc}
\mathrm{e}^{\mathrm{i}\left(x_{1}-x_{2}\right)} & \mathrm{e}^{\mathrm{i}\left(x_{1}-x_{2}\right)} \\
\mathrm{e}^{\mathrm{i}\left(x_{1}-x_{2}\right)} & 2 \cos \left(x_{1}-x_{2}\right)+\mathrm{e}^{-\mathrm{i}\left(x_{1}-x_{2}\right)}
\end{array}\right),
\end{aligned}
$$

where $I_{0}$ is a constant factor having the dimensions of an intensity. The BCP matrix is shift invariant, as was expected, and does not change upon propagation, giving rise, in particular, to uniform Stokes parameters (namely $I(x, z)=$ $\left.I_{0}, s_{1}(x, z)=-I_{0} / 2, s_{2}(x, z)=I_{0} / 2, s_{3}(x, z)=0\right)$ and degree of polarization $(p(x, z)=1 / \sqrt{2})$.

If a correlation is introduced between the two waves, interference phenomena occur for the Stokes parameters. Let us take, for instance, a correlation coefficient between the plane-wave amplitudes equal to $1 / \sqrt{2}$, so that the $\hat{A}_{n m}$ correlation matrices, evaluated from equation (12) and arranged themselves into a matrix form, turn out to be

$$
\left(\begin{array}{ll}
\hat{A}_{-1,-1} & \hat{A}_{-1,+1} \\
\hat{A}_{+1,-1} & \hat{A}_{+1,+1}
\end{array}\right)=\frac{I_{0}}{4}\left(\begin{array}{l}
\left(\begin{array}{ll}
0 & 0 \\
0 & 2
\end{array}\right)\left(\begin{array}{ll}
0 & 0 \\
1 & 1
\end{array}\right) \\
\left(\begin{array}{ll}
0 & 1 \\
0 & 1
\end{array}\right)\left(\begin{array}{ll}
1 & 1 \\
1 & 1
\end{array}\right)
\end{array}\right),
$$

thus leading to a BCP matrix of the form

$$
\begin{aligned}
\hat{J}\left(x_{1},\right. & \left.x_{2}, z\right) \\
& =\hat{J}\left(x_{1}, x_{2}, 0\right)=\hat{J}_{M}\left(x_{1}-x_{2}\right)+\hat{J}_{P}\left(x_{1}+x_{2}\right) \\
& =\frac{I_{0}}{4}\left(\begin{array}{cc}
\mathrm{e}^{\mathrm{i}\left(x_{1}-x_{2}\right)} & \mathrm{e}^{\mathrm{i}\left(x_{1}-x_{2}\right)} \\
\mathrm{e}^{\mathrm{i}\left(x_{1}-x_{2}\right)} & 2 \cos \left(x_{1}-x_{2}\right)+\mathrm{e}^{-\mathrm{i}\left(x_{1}-x_{2}\right)}
\end{array}\right)
\end{aligned}
$$

(a)

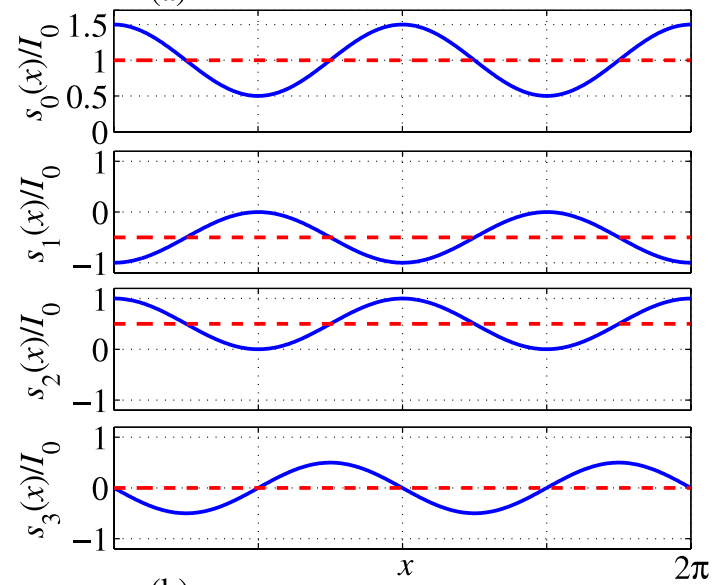

(b)

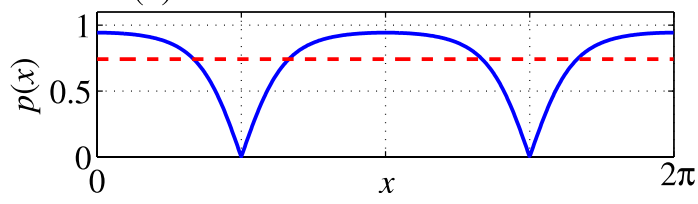

Figure 1. Stokes parameters (a) and degree of polarization (b) across any transverse plane for the field radiated by the source in equation (28) (dashed lines) and in equation (30) (solid lines).

$$
\begin{aligned}
& +\frac{I_{0}}{4}\left(\begin{array}{cc}
0 & \mathrm{e}^{\mathrm{i}\left(x_{1}+x_{2}\right)} \\
\mathrm{e}^{-\mathrm{i}\left(x_{1}+x_{2}\right)} & 2 \cos \left(x_{1}+x_{2}\right)
\end{array}\right) \\
& =\frac{I_{0}}{4}\left(\begin{array}{cc}
\mathrm{e}^{\mathrm{i}\left(x_{1}-x_{2}\right)} & 2 \mathrm{e}^{\mathrm{i} x_{1}} \cos x_{2} \\
2 \mathrm{e}^{-\mathrm{i} x_{2}} \cos x_{1} & \mathrm{e}^{-\mathrm{i}\left(x_{1}-x_{2}\right)}+4 \cos x_{1} \cos x_{2}
\end{array}\right),
\end{aligned}
$$

which does not depend on $z$. The corresponding polarization matrix is

$$
\hat{P}(x)=\frac{I_{0}}{4}\left(\begin{array}{cc}
1 & 2 \mathrm{e}^{\mathrm{i} x} \cos x \\
2 \mathrm{e}^{-\mathrm{i} x} \cos x & 3+2 \cos 2 x
\end{array}\right),
$$

and gives rise to the following Stokes vector:

$$
\mathbf{s}(x)=\frac{I_{0}}{2}\left(\begin{array}{c}
2+\cos 2 x \\
-1-\cos 2 x \\
1+\cos 2 x \\
-\sin 2 x
\end{array}\right)
$$

and degree of polarization

$$
p(x)=\sqrt{1-\frac{1}{(2+\cos 2 x)^{2}}} .
$$

Figure 1 shows the behavior of the Stokes parameters and the degree of polarization as functions of the transverse coordinate. Dashed lines represent the values of such parameters when the correlation between the two plane waves vanishes (corresponding to the BCP matrix in equation (28)). It can be seen that the correlation between the two component plane waves has the effect of introducing a modulation in all Stokes parameters and in the degree of polarization. 


\subsection{Three component plane waves}

Another significant example concerns a case where three component plane waves are involved. To this aim, let us consider a source with the following BCP matrix:

$$
\hat{J}\left(x_{1}, x_{2}, 0\right)=\frac{I_{0}}{4}\left(\begin{array}{cc}
1+\mathrm{e}^{\mathrm{i}\left(x_{1}-x_{2}\right)} & \mathrm{e}^{\mathrm{i} x_{1}}-\mathrm{e}^{\mathrm{i} x_{2}} \\
-\mathrm{e}^{-\mathrm{i} x_{1}}+\mathrm{e}^{-\mathrm{i} x_{2}} & 1+\mathrm{e}^{-\mathrm{i}\left(x_{1}-x_{2}\right)}
\end{array}\right) .
$$

As we shall see in the following, even in this case a physical model can be envisaged to physically produce a source with the above BCP matrix, so that the latter is surely bona fide.

The local polarization properties of the source are taken into account by the corresponding polarization matrix, that is

$$
\hat{P}(x, 0)=\frac{I_{0}}{2}\left(\begin{array}{ll}
1 & 0 \\
0 & 1
\end{array}\right),
$$

which denotes a completely unpolarized field profile with uniform transverse intensity.

The polarization matrix of the field radiated from such a source can be evaluated on computing the Fourier coefficients of the BCP matrix of the source, using equation (7). In this case it turns out that only the orders $-1,0$, and +1 contribute to the BCP matrix, and the $\hat{A}_{n m}$ correlation matrices are

$$
\begin{aligned}
& \left(\begin{array}{ccc}
\hat{A}_{-1,-1} & \hat{A}_{-1,0} & \hat{A}_{-1,+1} \\
\hat{A}_{0,-1} & \hat{A}_{0,0} & \hat{A}_{0,+1} \\
\hat{A}_{+1,-1} & \hat{A}_{+1,0} & \hat{A}_{+1,+1}
\end{array}\right)
\end{aligned}
$$

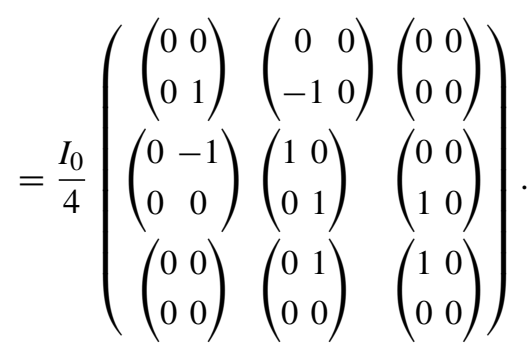

The values of the correlation coefficients denote that the -1 and the +1 orders are linearly polarized along $y$ and $x$, respectively, while the zero-order wave is completely unpolarized. Furthermore, the $y$ component of the order -1 is perfectly correlated (and in phase opposition) with the $x$ component of the order 0 , while the $x$ component of the order +1 is perfectly correlated with the $y$ component of the order 0 (and in phase). No correlation exists between the orders -1 and +1 .

This choice for the BCP matrix gives rise to a rather peculiar behavior of the polarization properties of the propagated field, as can be seen on evaluating the matrix $\hat{J}\left(x_{1}, x_{2}, z\right)$. From equation (15) we have indeed

$$
\begin{aligned}
& \hat{J}\left(x_{1}, x_{2}, z\right) \\
& \quad=\frac{I_{0}}{4}\left(\begin{array}{cc}
1+\mathrm{e}^{\mathrm{i}\left(x_{1}-x_{2}\right)} & \mathrm{e}^{\mathrm{i}\left(x_{1}-z\right)}-\mathrm{e}^{\mathrm{i}\left(x_{2}+z\right)} \\
-\mathrm{e}^{-\mathrm{i}\left(x_{1}+z\right)}+\mathrm{e}^{-\mathrm{i}\left(x_{2}-z\right)} & 1+\mathrm{e}^{-\mathrm{i}\left(x_{1}-x_{2}\right)}
\end{array}\right),
\end{aligned}
$$

and, on letting $x_{1}=x_{2}=x$, the polarization matrix takes the form

$$
\hat{P}(x, z)=\frac{I_{0}}{2}\left(\begin{array}{cc}
1 & -\mathrm{ie}^{\mathrm{i} x} \sin z \\
\mathrm{ie}^{-\mathrm{i} x} \sin z & 1
\end{array}\right) .
$$

Some considerations are worth making. First, the intensity turns out to be uniform (equal to $I_{0}$ ) within the whole half-space $z \geq 0$. Second, although the Stokes vector varies across the transverse planes according to the following relation:

$$
\mathbf{s}(x, z)=I_{0}\left(\begin{array}{c}
1 \\
0 \\
\sin x \sin z \\
\cos x \sin z
\end{array}\right),
$$

the degree of polarization, evaluated according to equation (5), i.e.,

$$
p(x, z)=|\sin z|,
$$

does not, but it changes periodically from zero to unity on varying $z$. This means that the field is completely unpolarized across the source plane $(z=0)$ but it becomes perfectly polarized at some distance from the source plane. More precisely, this happens at a distance equal to a quarter of the Talbot distance $(z=\pi / 2)$. This behavior may seem a little surprising, because the field at $z=0$ is perfectly undistinguishable from a single unpolarized plane wave, if only the local polarization characteristics of the two fields are taken into account (equation (35)). On the contrary, it should be kept in mind that the two fields are different as far as their two-point correlation properties are concerned (equation (34)).

Sources of the type described here are encountered, for instance, when a completely unpolarized plane wave impinges onto a double-wedge depolarizer (DWD), which is an optical device consisting of a pair of uniaxial crystal wedges with suitably oriented optic axes, placed in contact to form a plate (such as the model DPU-25-A produced by Thorlabs). In fact, following an analogous approach to the one used in [45], where the effects of the propagation on the polarization characteristics of a field produced in a similar case were investigated, it is possible to show that a DWD can be described by a transversely periodic Jones matrix of the following form:

$$
\hat{T}_{\operatorname{DWD}}(x)=\frac{1}{2}\left(\begin{array}{cc}
1+\mathrm{e}^{\mathrm{i} x} & -1+\mathrm{e}^{\mathrm{i} x} \\
1-\mathrm{e}^{-\mathrm{i} x} & 1+\mathrm{e}^{-\mathrm{i} x}
\end{array}\right) .
$$

To obtain equation (41), the DWD has been supposed to be oriented in such a way that the two edges, as well as the optical axis of the second crystal, are aligned along the $y$ axis. Furthermore, the transverse period for a real device depends on the refractive indices of the crystals employed and on the wedge angle, while we are using normalized coordinates, such that the period is taken as $2 \pi$.

When a completely unpolarized plane wave with intensity $I_{0}$ impinges onto such a device, the $\mathrm{BCP}$ matrix of the 
emerging field is

$$
\begin{aligned}
\hat{J}\left(x_{1}, x_{2}, 0\right) & =\frac{I_{0}}{2} \hat{T}_{\mathrm{DWD}}\left(x_{1}\right)\left(\begin{array}{ll}
1 & 0 \\
0 & 1
\end{array}\right) \hat{T}_{\mathrm{DWD}}^{\dagger}\left(x_{2}\right) \\
& =\frac{I_{0}}{4}\left(\begin{array}{cc}
1+\mathrm{e}^{\mathrm{i}\left(x_{1}-x_{2}\right)} & \mathrm{e}^{\mathrm{i} x_{1}}-\mathrm{e}^{\mathrm{i} x_{2}} \\
-\mathrm{e}^{-\mathrm{i} x_{1}}+\mathrm{e}^{-\mathrm{i} x_{2}} & 1+\mathrm{e}^{-\mathrm{i}\left(x_{1}-x_{2}\right)}
\end{array}\right),
\end{aligned}
$$

and is exactly of the form in equation (34).

A somewhat similar behavior appears at the output of a polarization grating (PG) [24] when it is orthogonally illuminated by an unpolarized plane wave having intensity $I_{0}$. In fact, the Jones matrix of a PG is [42]

$$
\hat{T}_{\mathrm{PG}}(x)=\left(\begin{array}{cc}
\cos ^{2} x & \sin x \cos x \\
\sin x \cos x & \sin ^{2} x
\end{array}\right),
$$

and, on proceeding as for the previous case, the polarization matrix at a typical transverse plane turns out to be

$$
\begin{aligned}
& \hat{P}(x, z) \\
& \quad=\frac{I_{0}}{4}\left(\begin{array}{cc}
1+\cos (2 x) \cos (4 z) & \sin (2 x) \cos (4 z) \\
\sin (2 x) \cos (4 z) & 1-\cos (2 x) \cos (4 z)
\end{array}\right) .
\end{aligned}
$$

In this case, too, the intensity is uniform in the whole half-plane $z \geq 0$ and the degree of polarization is uniform across the transverse plane, but the latter oscillates along the $z$ direction as

$$
p(x, z)=|\cos (4 z)|,
$$

passing from 1 to 0 when $z$ goes from 0 to one eighth of the Talbot distance.

\subsection{More than three component plane waves}

More complex and general field structures are obtained on combining more than three plane waves. This happens, for instance, when the field described by the BCP matrix in equation (28) impinges onto an isotropic sinusoidal amplitude grating characterized by a Jones matrix of the form

$$
\hat{T}_{\mathrm{SG}}(x)=\sin ^{2}(x / 2)\left(\begin{array}{ll}
1 & 0 \\
0 & 1
\end{array}\right) \text {. }
$$

It can be verified that the resulting source consists of five mutually correlated plane waves. The evaluation of the polarization matrix for the propagated field leads, in particular, to the distributions of the intensity and the degree of polarization across the plane $x z$ shown in figure 2 . It can be noticed that both of them depend on both $x$ and $z$, and present the periodicities predicted by the general theory of section 3 .

\section{Conclusions}

A plane-wave decomposition has been used to study the polarization properties of the field radiated from partially coherent planar electromagnetic sources with periodic variation of their beam coherence-polarization matrix. Within the paraxial approximation, the transverse periodicity of the

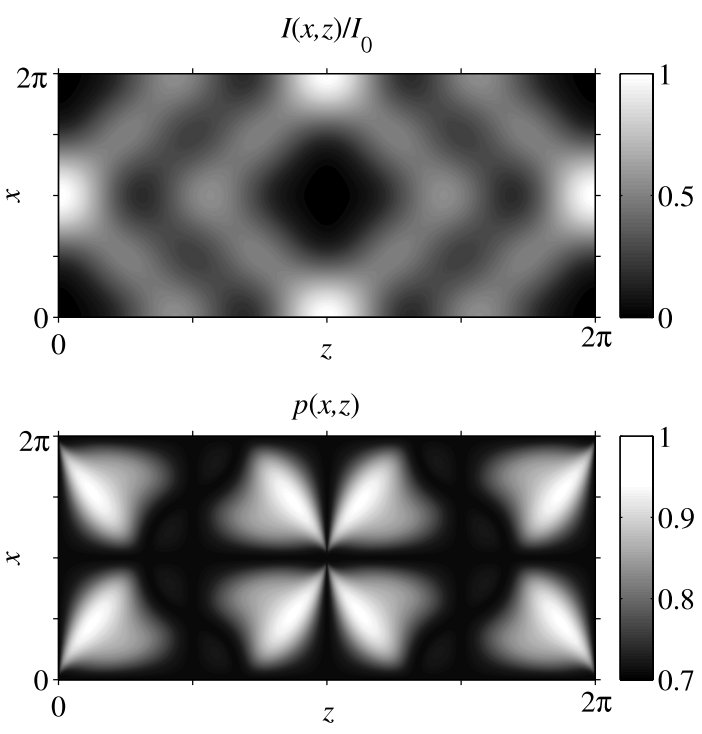

Figure 2. The intensity and degree of polarization for the source given in equation (28) after passing through an amplitude grating described by the Jones matrix in equation (46).

source yields a longitudinal periodicity of the polarization features of the propagated field, thus representing an extension of the Talbot effect to the case of partially coherent electromagnetic sources. In particular, conditions under which a periodic BCP matrix remains invariant upon paraxial propagation have been obtained.

Several examples of sources of this kind have been presented, together with experimental procedures to synthesize them, and different behaviors of the propagated fields have been shown. For instance, it has been evidenced that completely unpolarized sources with a uniform intensity profile can be realized, for which the propagated field becomes perfectly polarized across some transverse planes. The opposite situation, i.e., totally polarized sources for which the propagated field becomes completely unpolarized at certain planes, is also achievable.

\section{References}

[1] Talbot H F 1836 Facts relating to optical science Phil. Mag. $9401-7$

[2] Lohmann A W and Silva D E 1971 An interferometer based on the Talbot effect Opt. Commun. 2 413-5

[3] Patorski K 1989 The self-imaging phenomenon and its applications Progress in Optics vol 27, ed E Wolf (Amsterdam: North-Holland) pp 3-108

[4] Noponen E and Turunen T 1992 Electromagnetic theory of Talbot imaging Opt. Commun. 98 132-40

[5] Oreb B F and Dorsch R G 1994 Profilometry by phase-shifted Talbot images Appl. Opt. 33 7955-62

[6] Arrizón V, Tepichin E, Ortiz-Gutiérrez M and Lohmann A W 1996 Fresnel diffraction at 1/4 of the Talbot distance of an anisotropic grating Opt. Commun. 127 171-5

[7] Wei S, Wu S, Kao I and Chiang F P 1998 Measurement of wafer surface using shadow moiré technique with Talbot effect J. Electron. Packag. 120 166-70

[8] Tebaldi M, Rueda P J E and Bolognini N 2000 Talbot interferometer based on a birefringence grating $\mathrm{Opt}$. Commun. 185 65-76 
[9] Tervo J and Turunen J 2001 Transverse and longitudinal periodicities in fields produced by polarization gratings Opt. Commun. 190 51-7

[10] Bomzon Z, Niv A, Biener G, Kleiner V and Hasman E 2002 Polarization Talbot self-imaging with computer-generated, space-variant subwavelength dielectric gratings Appl. Opt. $415218-22$

[11] Schirripa-Spagnolo G, Ambrosini D and Paoletti D 2002 Displacement measurement using the Talbot effect with a Ronchi grating J. Opt. A: Pure Appl. Opt. 4 S376-80

[12] Zhang Y, Yao X, Yuan C, Li P, Yuan J, Feng W, Jia S and Zhang Y 2012 Controllable multiwave mixing Talbot effect IEEE Photon. J. 4 2057-65

[13] Lau E 1948 Beugungserscheinungen an Doppelrastern Ann. Phys. 6 417-23

[14] Gori F 1983 Lau effect and coherence theory Opt. Commun. 31 4-8

[15] Lohmann A W and Ojeda-Castañeda J 1983 Spatial periodicities in partially coherent fields Opt. Acta: Int. J. Opt. 30 475-9

[16] Indebetouw G 1984 Propagation of spatially periodic wavefields Opt. Acta: Int. J. Opt. 31 531-9

[17] Liu L 1988 Partially coherent diffraction effect between Lau and Talbot effects J. Opt. Soc. Am. A 5 1709-16

[18] Turunen J, Vasara A and Friberg A T 1991 Propagation invariance and self-imaging in variable-coherence optics J. Opt. Soc. Am. A 8 282-9

[19] Lohmann A W, Mendlovic D and Shabtay G 1998 Talbot (1836), Montgomery (1967), Lau (1948) and Wolf (1955) on periodicity in optics Pure Appl. Opt. 7 1121-4

[20] Teng S, Liu L, Zu J, Luan Z and Liu D 2003 Uniform theory of the Talbot effect with partially coherent light illumination J. Opt. Soc. Am. A 20 1747-54

[21] Jahns J and Lohmann A W 2009 Optical wavefields with lateral and longitudinal periodicity Appl. Opt. 48 3438-45

[22] Gori F, Santarsiero M, Vicalvi S, Borghi R and Guattari G 1998 Beam coherence-polarization matrix Pure Appl. Opt. 7 941-51

[23] Wolf E 2003 Unified theory of coherence and polarization of random electromagnetic beams Phys. Lett. A 312 263-7

[24] Gori F, Santarsiero M, Borghi R and Piquero G 2000 Use of the van Cittert-Zernike theorem for partially polarized sources Opt. Lett. 25 1291-3

[25] Agrawal G P and Wolf E 2000 Propagation-induced polarization changes in partially coherent optical beams J. Opt. Soc. Am. A 17 2019-23

[26] Gori F, Santarsiero M, Piquero G, Borghi R, Mondello A and Simon R 2001 Partially polarized Gaussian Schell-model beams J. Opt. A: Pure Appl. Opt. 3 1-9

[27] Piquero G, Gori F, Romanini P, Santarsiero M, Borghi R and Mondello A 2002 Synthesis of partially polarized Gaussian Schell-model sources Opt. Commun. 208 9-16

[28] Tervo J 2003 Azimuthal polarization and partial coherence J. Opt. Soc. Am. A 20 1974-80

[29] Gori F, Santarsiero M, Borghi R and Wolf E 2006 Effects of coherence on the degree of polarization in a Young interference pattern Opt. Lett. 31 688-90
[30] Martínez-Herrero R and Mejías P M 2007 Electromagnetic fields that remain totally polarized under propagation $O p t$. Commun. 279 20-2

[31] Wolf E 2007 Polarization invariance in beam propagation Opt. Lett. 32 3400-1

[32] Salem M and Wolf E 2008 Coherence-induced polarization changes in light beams Opt. Lett. 33 1180-2

[33] Gori F 2008 Partially correlated sources with complete polarization Opt. Lett. 33 2818-20

[34] Gori F, Tervo J and Turunen J 2009 Correlation matrices of completely unpolarized beams Opt. Lett. 34 1447-9

[35] Martínez-Herrero R, Mejías P M and Piquero G 2009 Characterization of Partially Polarized Light Fields (Springer Series in Optical Sciences vol 147) (Berlin: Springer-Verlag)

[36] Visser T D, Kuebel D, Lahiri M, Shirai T and Wolf E 2009 Unpolarized light beams with different coherence properties J. Mod. Opt. 56 1369-74

[37] Martínez-Herrero R and Mejías P M 2010 On the propagation of random electromagnetic fields with position-independent stochastic behavior Opt. Commun. 283 4467-9

[38] Ramírez-Sánchez V, Piquero G and Santarsiero M 2010 Synthesis and characterization of partially coherent beams with propagation-invariant transverse polarization pattern Opt. Commun. 283 4484-9

[39] Zhang R, Wang X, Cheng X and Qiu Z 2010 Polarization distribution control of anisotropic electromagnetic Gaussian Schell-model beams on free propagation by exploiting correlation properties at the source plane J. Opt. Soc. Am. A 27 2496-505

[40] Vidal I, Fonseca E J S and Hickmann J M 2011 Light polarization control during free-space propagation using coherence Phys. Rev. A 84033836

[41] Tong Z and Korotkova O 2012 Electromagnetic nonuniformly correlated beams J. Opt. Soc. Am. A 29 2154-8

[42] Gori F 1999 Measuring Stokes parameters by means of a polarization grating Opt. Lett. 24 584-6

[43] Piquero G, Borghi R and Santarsiero M 2001 Gaussian Schell-model beams propagating through polarization gratings J. Opt. Soc. Am. A 18 1399-405

[44] Piquero G, Borghi R, Mondello A and Santarsiero M 2001 Far field of beams generated by quasi-homogeneous sources passing through polarization gratings Opt. Commun. $195339-50$

[45] de Sande J C G, Santarsiero M, Piquero G and Gori F 2012 Longitudinal polarization periodicity of unpolarized light passing through a double wedge depolarizer Opt. Express $2027348-60$

[46] Wolf E 2007 Introduction to the Theory of Coherence and Polarization of Light (Cambridge: Cambridge University Press)

[47] Gori F, Guattari G, Palma C and Padovani C 1988 Specular cross-spectral density functions Opt. Commun. $68239-43$

[48] Setälä J, Tervo J and Friberg A T 2006 Contrasts of Stokes parameters in Young's interference experiment and electromagnetic degree of coherence Opt. Lett. 31 2669-71 